\title{
Monitoring cytokine profiles during immunotherapy
}

Measuring cytokine production is an integral part of measuring immune response during immunotherapy. Current technologies allow the simultaneous quantification of multiple cytokines in a variety of tissues. Patterns of cytokine response can be referred to as cytokine profiles. This article discusses the experimental design and data analysis of a number of studies that examined cytokine profiles in humans. We highlight potential sources of variability, both due to assay nuances and the diversity of human populations. We present strategies for analyzing data, emphasizing both multidimensional analysis and the value of treating each donor as his or her own control.

\section{KEYWORDS: cytokine profile data analysis data mining immune monitoring immunotherapy}

Cytokines are small proteins secreted by various cells of the immune system that provide cell to cell signaling, usually within a microenvironment. Measuring cytokine production is an integral part of measuring immune response [1]. Monitoring cytokine profiles during immunotherapy can provide insights into changes in the immune system and useful biomarkers of early response to treatment [2]; and subsequently indicators to guide the course of treatment and adaptive clinical trial design [3]. The primary goal of this article is to review the existing literature that can inform the design and analysis of the next generation of cytokine monitoring studies. Herein, we summarize a variety of studies on cytokine monitoring in humans, discussing how authors have analyzed and presented results, with an emphasis on studies measuring a large number of cytokine readouts. In addition, we highlight some of the challenges of monitoring cytokine profiles in diverse human populations and identify multiple possible sources of physiological variability. Finally, we provide some thoughts about the design of future studies.

The studies discussed here are drawn primarily from cancer immunotherapy research, with a few examples from studies of transplantation, autoimmunity and infectious disease vaccine response. In addition, several studies in aging and stress research were included owing to the large number of patients and/or the large number of cytokines described. To be included, studies needed to consider at least two cytokines. While there is also a body of literature on monitoring cytokines in allergy [4-6], these studies are not discussed here. We did not attempt to interpret or generalize the biological findings described in the included studies, in part because they are diverse in terms of disease, therapy, assays and tissues, but more importantly because our focus is on the experimental design of the studies and the analysis of results.

\section{An overview of monitoring}

TABLEs 1 \& 2 summarize studies of cytokine production in a variety of settings, including immunotherapy. Table 1 focuses on measurement studies, which by our definition include data for only one time point, while Taвцe 2 focuses on monitoring studies, which include at least two time points. Both tables include the number of donors, the number of cytokines/chemokines measured, the tissue(s) analyzed, the technology platform(s) used to collect the data, the disease of interest and the year of publication. The largest number of donors considered here is 738 .

None of these studies approach the scale of large long-term monitoring studies of autoimmunity in terms of number of donors and number of time points. For example, the Diabetes Autoimmunity Study in the Young (DAISY) has followed over 2500 children for up to 16 years [Barriga K, Pers. Сomm.]. Children are tested at 9, 15 and 24 months of age, and annually thereafter for the presence of autoantibodies. Duplicate samples are analyzed, and readouts are classified as positive when the mean of the duplicates is greater than the 99th percentile of the control population. Positive results are confirmed in such a way that a positive classification is based on at least four positive tests. In addition, $5 \%$ of negative samples are blinded and

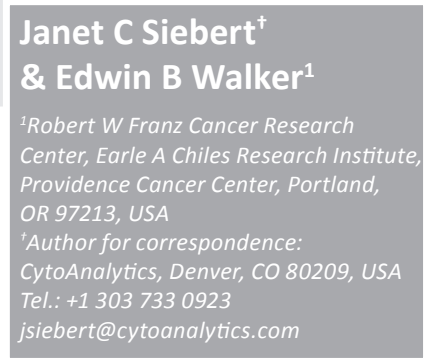


Table 1. Summary of measurement studies.

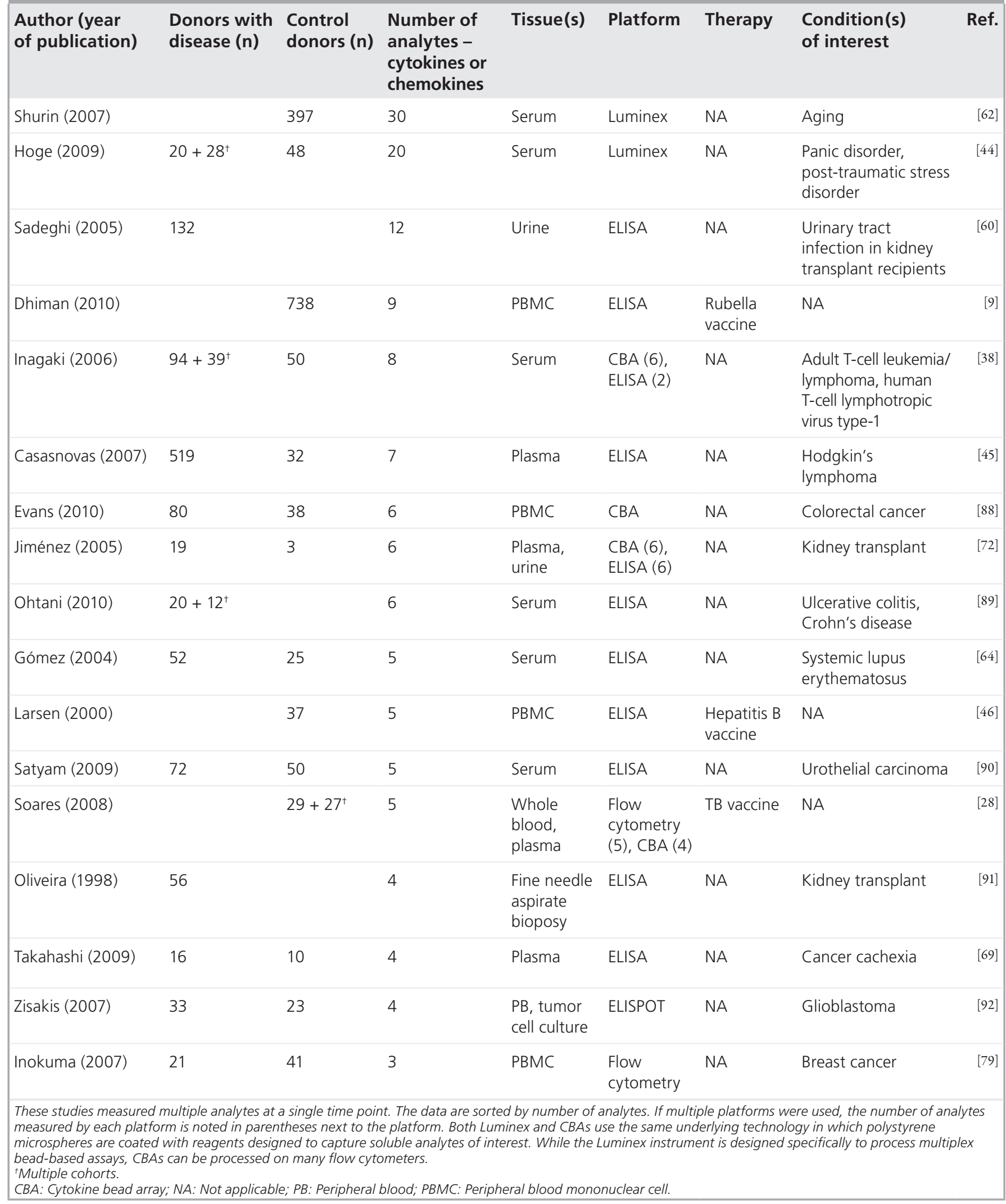




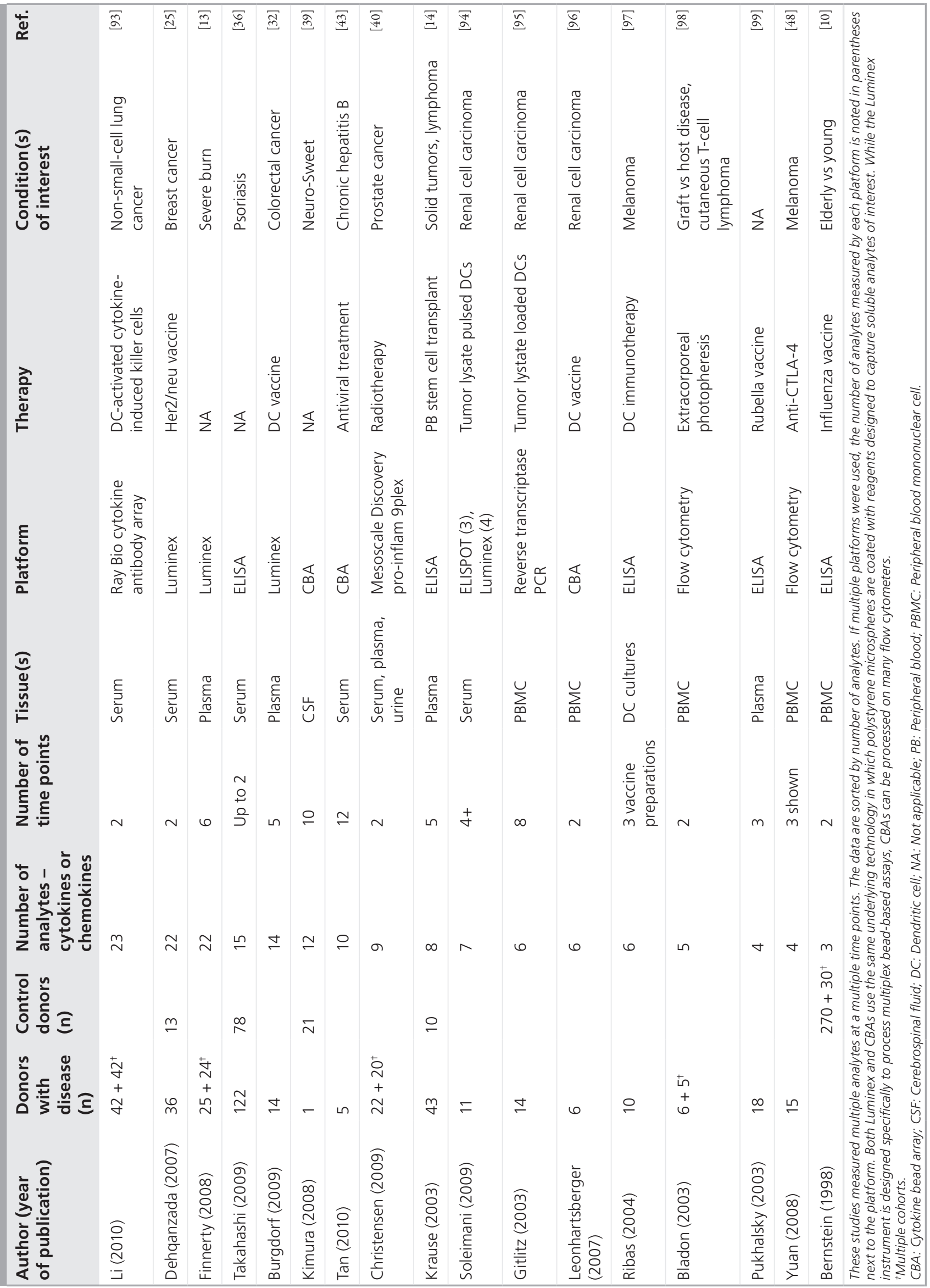




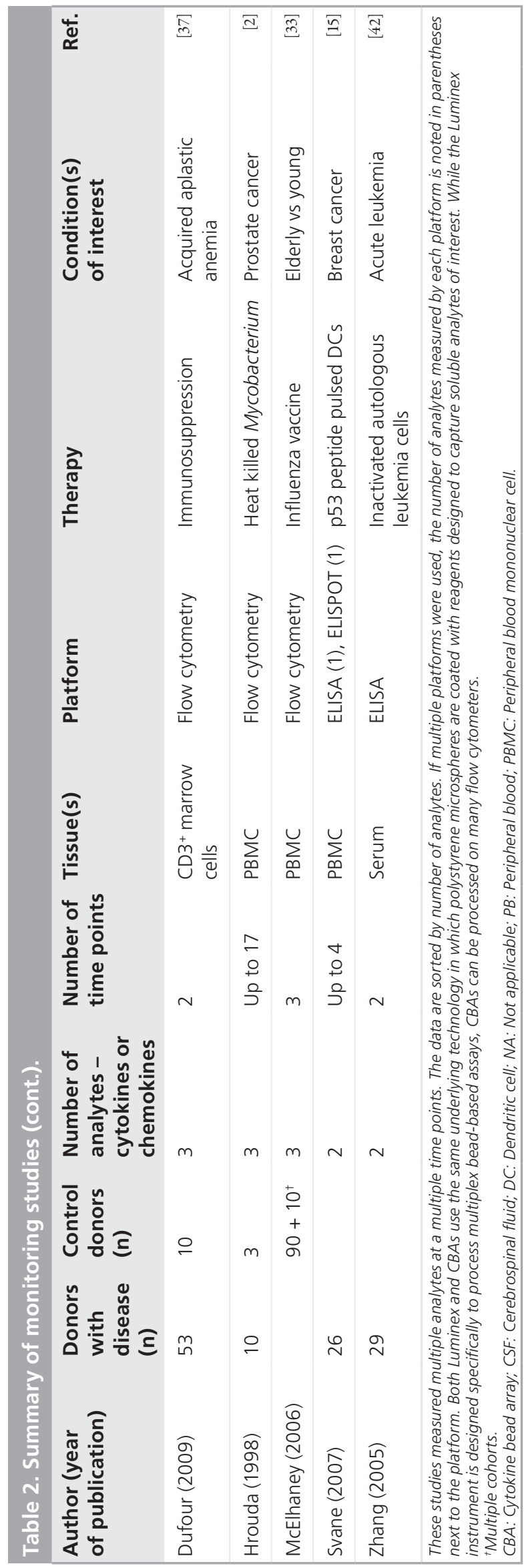

retested [7]. Related work has shown that the number of positive antibodies is predictive of risk [8]. Among first-degree relatives of patients with type 1 diabetes, the 5 -year risk of diabetes was $15 \%$ if one autoantibody was positive, $44 \%$ if two were positive, and $100 \%$ if three were positive, illustrating the importance of monitoring more than one biomarker. The design of and observations gleaned from such large-scale long-term studies can inspire better monitoring studies associated with immunotherapy. While cancer or transplantation immunotherapy studies may not be able to follow patients for 20 years, the number of patients, the number of time points, and therefore the rigor with which positivity are evaluated are all instructive.

In addition, infectious disease vaccine studies can provide valuable reference data sets for immunotherapy studies. In contrast to experimental cancer therapies or transplantation, vaccination for rubella [9] or influenza [10] provides a large patient pool (738 and 300, respectively) of presumably healthy donors. Immune responses to these vaccines are relatively robust, particularly as compared with tumor-associated antigens. Thus, documented cytokine responses to infectious disease vaccines help to calibrate a strong response in normal healthy donors. Knowledge of these cytokine response patterns can inform the interpretation of immune functional response patterns in potentially immunocompromised donors, such as the response of cancer patients to more weakly immunogenetic self-tumor antigens.

Interestingly, the Cancer Vaccine Clinical Trial Working Group recommended that proofof-principle trials should include a minimum of 20 patients, and that immune response be demonstrated by two separate assays at two consecutive time points after initial baseline assessment (three time points in total) [11]. In addition, Millán et al. speak to the value of measuring both specific analytes and general measurements of immune response such as lymphocyte activation and proliferation [12]. While the studies reviewed here were not necessarily designed as proof-of-principle trials, only three of the 24 monitoring studies summarized in TABLE 2 studied at least 20 patients for at least three time points [13-15]. Two of these included multiple immune response assays [14,15].

\section{Cytokine milieus}

Because cytokines act in networks, monitoring of a single cytokine may be of limited use [16]. In addition, cytokines have overlapping functions. Asking the question 'What cytokines are being 
produced and how is that production changing over time?' is fundamentally different from asking the question 'Is IFN- $\gamma$ being produced?' Additionally, there is risk in measuring one or two cytokines and generalizing that the response is Th1 or Th2, particularly if the signal cannot be associated with the particular cell population that is generating it. van den Engel et al. measured IFN- $\gamma$ and IL-5 production in cultures of tumor-infiltrating lymphocytes and lymph nodes, and concluded that such cultures showed mixed type 1/type 2 responses [17]. Kyte and colleagues measured secretion of six cytokines from bulk T-cell cultures from long-term cancer survivors after peptide vaccination [18]. Resulting cytokine profiles were IFN- $\gamma^{\text {high }} / \mathrm{IL}-10^{\text {low }} / \mathrm{IL}-4^{\text {low }}$ but also IL- $5^{\text {high }} / \mathrm{IL}-13^{\text {high }}$, and thus not easily classified as Th1 or Th2. The authors observed that while IFN- $\gamma$ is frequently employed as the only indicator of cytokine response in clinical trials, the overall cytokine milieu characterized by the measurement of multiple cytokines may be more informative. Interestingly, Dhiman and colleagues reported that 644 of $713(90.3 \%)$ children (median age: 15) vaccinated for rubella showed a positive response for IFN- $\gamma$ while $643(89.9 \%)$ showed a positive response for IL-10 [9]. Consequently, most of the children produced both Th1 and Th2 responses. Additionally, 99\% showed extremely strong IL-6 responses, with a median response of $3681 \mathrm{pg} / \mathrm{ml}$, calculated as the readout from the stimulated aliquot minus the readout from the unstimulated aliquot. Thus, measuring IFN- $\gamma$ alone does not adequately capture the full range of cytokine profiles.

Evidence of cytokine storms offers another argument for measuring multiple cytokines. Data collected in association with the wellknown Phase I trial of the anti-CD28 monoclonal antibody TGN1412 showed sudden and dramatic post-treatment increases in nine cytokines [19]. Subsequent work has shown that ANC28, a commercially available mitogenic CD28 antibody, can induce the secretion of multiple inflammatory cytokines (IL-2, IL-8, TNF- $\alpha$ and IFN- $\gamma$ ) without T-cell receptor coligation or cross-linking [20].

Demonstrations of plasticity, coupled with increasing documentation of multiple T-cell subsets (e.g., Th17, Th9 and Th22), also illustrate the complexity of the cytokine network and modulating feedback loops [21-23]. van der Vliet and colleagues demonstrated that NKT cells could be polarized to produce IFN- $\gamma$ or IL- 4 depending on whether they were cultured with
IL-15 or IL-7, respectively. In addition, polarization could be reversed by changing the culture conditions, indicating plasticity of the cells [21]. Thus, in vitro findings associated with culture conditions suggest that the cytokine milieu of the in vivo microenvironment may be an important aspect of the cytokine network. Furthermore, cancer involves multiple molecular alterations and potentially destabilized pathways, the detection of which might require the measurement of as many readouts as possible [24]. Consequently, broad-based monitoring of the cytokine milieu may be integral to future studies.

\section{Platforms \& applications}

Technologies used to measure cytokines include ELISA, enzyme-linked immunosorbent spot (ELISPOT), multiplex cytokine bead arrays (CBAs; Luminex or other), intracellular cytokine staining (ICS) by flow cytometry and gene expression platforms. CBAs allow the rapid analysis of multiple analytes in small volumes of specimens (e.g., $25 \mu \mathrm{l}$ ) [25]. Both Luminex and CBAs use the same underlying technology in which polystyrene microspheres are coated with reagents designed to capture soluble analytes of interest. While the Luminex instrument is designed specifically to process multiplex bead-based assays, CBAs can be processed on many flow cytometers. Hereafter, both techniques will be referred to as CBAs. ELISA and CBAs are bulk assays in which the overall concentration of a cytokine can be measured in a liquid phase sample such as serum, plasma or urine, or supernatant from cultured cells. Both ELISPOT and ICS detect cytokine production at a single-cell level. While ELISPOT readouts are generally limited to a single cytokine per well, measuring two cytokines is technically feasible [26]. In addition, with ELISPOT, there is no ability to discern phenotype or lineage of the spot-forming cells, other than through prior knowledge of the phenotype of the cells that were placed in the well. ICS supports the simultaneous interrogation of multiple cytokines, coupled with lineage markers such as CD3, CD4 and CD8, and/or memory/effector phenotype markers such as CCR7, CD57, CD27 and CD45RO [27,28]. However, the number of cytokines that can be simultaneously measured is less than with CBA. Two sources compare ELISPOT and ICS, discussing nuances of both assays $[29,30]$. Gene expression platforms support the simultaneous quantification of mRNA of multiple cytokines. However, due to post-translational modifications, mRNA levels may not be consistent with protein expression [31]. 
Burgdorf et al. used a 15-plex kit on a Luminex platform to measure cytokines and chemokines in patients receiving a dendritic cell vaccine $(n=14)$. Patients were stratified by progressive or stable disease. Analytes that changed significantly over the course of the trial (up to 120 days) were identified. In general, for those patients with stable disease, cytokine levels increased at 30 and 60 days, and decreased thereafter [32]. Dehqanzada et al. used a 22-plex kit on a Luminex platform to assess serum cytokine profiles in healthy controls and Her $2 /$ neu-expressing breast cancer patients. $H L A-A^{*} 02$-positive patients were treated with an $\mathrm{E} 75$ peptide vaccine. The authors identified significant differences in cytokine levels in sera of patients compared with controls, of nodenegative compared with node-positive patients, and in pre- and post-vaccination responses of vaccinated patients [25]. This data suggests that such differences in cytokine profiles can be used in screening, diagnosis and staging of patients.

A study of intracellular cytokine expression in response to influenza vaccination measured IFN- $\gamma$, IL-10 and TNF- $\alpha$ in 90 elderly adults and ten young adult controls at three time points using flow cytometry. Nine elderly adults who subsequently developed laboratory-diagnosed influenza (LDI) were classified as LDI while the other elderly adults were non-LDI. The data showed tenfold lower levels of IFN- $\gamma$ and threefold higher levels of IL-10 in the LDI group than in the non-LDI group. The data also showed that in influenza-specific $\mathrm{CD}^{+}$and $\mathrm{CD}^{+}$cells stimulated with live influenza virus and IL-7, more than $90 \%$ of the $\mathrm{CD}^{6} 9^{+}$granzyme $\mathrm{B}^{+}$cells were also IFN- $\gamma$ and IL-10 double-positive, suggesting again that the Th1/Th2 distinction may not be relevant in this context. In addition, the authors concluded that such T-cell cytokine response measurements provide better correlation of vaccine efficacy than do serum antibody levels [33].

A study of bacillus Calmette-Guérin tuberculosis vaccine in newborns measured intracellular cytokine levels of IFN- $\gamma$, IL-2, TNF- $\alpha$, IL- 4 and IL-10 in $\mathrm{CD}^{+}$and $\mathrm{CD}^{+} \mathrm{T}$ cells. Memory phenotypes were further characterized with CCR7, CD45RA and CD27, in a panel including IFN- $\gamma$ and IL-2. Both analyses used flow cytometry. The authors noted diverse cytokine repertoires, with some infants having cells that produced mostly one of the measured cytokines, while others had cells that produced mostly three cytokines. In aggregate results, the data showed multiple cell subsets as defined by cytokine profiles. Interestingly, for all donors $(\mathrm{n}=29)$, more cells expressed TNF- $\alpha$ and/or IL-2 than IFN- $\gamma$ alone. The authors commented that measuring a single cytokine such as IFN- $\gamma$ does not properly characterize the magnitude or the complexity of the immune response [28]. Multiple studies suggest that such multiparameter cytokine profiles may prove to be an important indicator of immune response [27,34,35].

In summary, a variety of platforms can be used to monitor cytokine profiles during immunotherapy. The applications discussed above illustrate larger studies, both in terms of number of donors and number of analytes. While multiplex assays allow interrogation of a large number of analytes using a small sample volume, the results are not specific to a particular cell lineage unless specific cells were selected as part of the protocol. In contrast, flow cytometry assays support the simultaneous identification of lineage phenotypes, sublineage phenotypes such as memory and effector $\mathrm{T}$ cells, and correlated intracellular cytokine production at a single-cell level.

\section{Experimental design \\ - Control constructs}

In the studies reviewed here, there were five main approaches to defining control populations or samples. They were comparing donors with disease to healthy controls $[25,36,37]$, comparing donors with one disease to donors with another disease $[38,39]$, comparing donors with one disease state to another (e.g., stable disease to progressive disease) [25,32], comparing pre-treatment readouts to post-treatment readouts [40-42], and using the individual donor as his or her own control over three or more time points [43].

\section{Defining the readout}

In general, the readout for liquid-phase assays such as ELISA, Luminex and CBAs is a measure of concentration, such as picograms per milliliter $(\mathrm{pg} / \mathrm{ml})$. Some authors reported the mean of duplicates $[44,45]$. Others reported a 'baselinesubtracted' value, such as cytokine concentration in response to sample stimulation with antigen, after subtraction of the background level in a parallel unstimulated culture [46]. In a combination of these two approaches, Ovsyannikova and colleagues performed triplicate measures both prior to stimulation and after stimulation, reporting a single readout based on median of stimulated minus median of unstimulated [47]. In ICS assays, readouts can be reported as the percentage of responding cells or the absolute number of responding cells. Some authors subtracted the corresponding response in negative controls [27]. 


\section{Positive cutoffs}

Using a stringent definition for positive readouts, Kimura et al. compared their one patient with Neuro-Sweet disease to 21 controls having other neurological disorders. They considered a readout positive if it exceeded the mean plus three standard deviations (SD) of the readout in the control group [39]. At the other end of the spectrum, Dhiman and colleagues defined positive as simply the post-treatment value exceeding the pre-treatment value [9]. Svane et al. defined positive responses as greater than two times the pre-vaccine level [15]. Yuan et al. defined positive as at least one post-therapy sample being greater than three times the corresponding pre-therapy sample, and consisting of at least $0.1 \%$ of responding cells [48]. Similarly, Horton et al. defined a cytokine response as positive if the antigen-specific response was at least three times greater than the negative control, and at least $0.05 \%$ above that control [49]. Comin-Anduix et al. argued that immunological response should be determined in one of two ways, either donor-specific movement from below the lower limit of detection (LLD) to above the LLD, or movement beyond an assay-specific reference change value for those donors whose baseline measurement was above the LLD [50]. Collectively, these works demonstrate that there are multiple approaches to defining control populations, assay readouts and positive immune response that can be employed depending on experimental design and the questions being asked in the study.

\section{Assay optimization, standardization \& variability}

A number of studies have addressed the topics of assay development, optimization and standardization [16,31,49,51-54]. In addition, the MIATA Project (Minimum Information About T cell Assays) is an effort to articulate and encourage the capture of information required for the objective assessment of the quality of such assays [55]. Taken together, these works provide a valuable body of literature for anyone wanting to optimize and validate a multiparameter cytokine monitoring assay.

In a study of reliability and reproducibility of a multiplex assay for cytokine measurements, de Jager $e t$ al. showed that while cytokine measurements are stable for up to 2 years when stored at $-80^{\circ} \mathrm{C}$, they do not remain stable after repeated freeze-thaw cycles [16]. Liu et al. demonstrated that simultaneous detection of 17 cytokines in undiluted whole blood is a viable approach to detecting immune status. They showed kinetics and dose-dependent effects of three different immunosuppressants on multiple cytokines.
They also showed that different immunosuppressants drive distinct cytokine profiles. In addition, they suggested that an assay using undiluted whole blood better mimics the in vivo environment than assays involving isolation and culture procedures [31]. However, other researchers have shown that IFN- $\gamma$ readouts, as measured by flow cytometry, are higher in peripheral blood mononuclear cells (PBMCs) than in whole blood, perhaps because whole blood contains plasma proteins that may interfere with antigen uptake or MHC loading [56]. Other authors discussed multicenter efforts to harmonize ELISPOT results [53,54].

Nomura and colleagues provided a detailed yet easy-to-read discussion of the issues and strategies involved in developing multiparameter ICS assays [51]. Maecker $e t$ al. discussed cross-site reproducibility of such assays [52]. Both studies demonstrated that the use of lyophilized reagents can reduce variability due to reagent batch and/or reagent deterioration. In addition, inter-operator variation in gating can be reduced by having a single operator perform all gating. Using centralized single-operator gating and lyophilized reagents, inter-laboratory coefficients of variation (CVs) were less than $20 \%$, even for relatively low response levels (e.g., $0.1-1.5 \%$ of cytokine positive $\mathrm{CD}^{+}{ }^{+}$or $\mathrm{CD} 8{ }^{+}$cells) [52].

Horton et al. detailed the validation of an eight color ICS assay that simultaneously measured IFN- $\gamma$, IL-2, IL- 4 and TNF- $\alpha$ by CD $4^{+}$ and $\mathrm{CD}^{+} \mathrm{T}$ cells. They considered such factors as linearity, precision, sensitivity, specificity and detection limits per FDA Center for Drug Evaluation and Research guidance on Bioanalytical Method Validation. Their assay was organized into sets of steps, each of which could be completed within an 8-hour workday. One particularly important finding was that using a viability dye avoids the overestimation of antigen-specific immune response. In addition, they captured inter- and intra-sample, inter-day and inter-operator CVs. They defined the limit of quantification (LOQ) of their assay as the lowest frequency of antigen-specific T-cell response for which the CV was $30 \%$ or less. These LOQs ranged from near zero to $0.08 \%$ [49].

One recommendation of the International Society for Biological Therapy in Cancer-FDA taskforce on immunotherapy biomarkers was that analytical variation of an assay should be determined and that CVs should be reported [57,101]. Another working group commented that the maximum justifiable amount of sample should be collected to permit retesting [11]. However, 
it is often the case that small sample size limits the amount of possible repeat testing. This problem can be addressed in part by the inclusion of standardized, validated performance controls with the analysis of each set of patient samples. Frozen defined serum or culture media analyte standards can be employed in ELISA and CBA assays, while cryopreserved cells with known stimulated cytokine expression profiles can be used as standardized performance controls for ICS assays. Reasonable and expected results from such performance control samples minimally confirm that the assay was run reproducibly and meets the established criteria for an accurate assay performance. Clearly, calculating and understanding assay precision is essential to understanding positive responses.

While the articles discussed above specifically address assay optimization and standardization, some traditional research studies mention and/or try to control factors that can impact variability. Diurnal effects, absolute cell count and the kinetics of cytokine production can all impact assay variability. One study noted that the majority of samples were taken between 8 a.m. and 9 a.m. [44]. Another study, which measured cytokines in cerebrospinal fluid, noted that the concentration of the cytokines was correlated with absolute cell count [39]. Suntharalingam et al. showed the kinetics of nine cytokines over a 5-day period after infusion of anti-CD28 monoclonal antibody. Median levels of all cytokines peaked within $24 \mathrm{~h} \mathrm{[19].} \mathrm{Strong} \mathrm{responses}$ associated with immunotherapeutic vaccines may take longer to develop. Carbone et al. documented that IFN- $\gamma$ responses of one patient, after repeated immunization with p53- and K-ras-derived peptides, were barely detectable at weeks 0 and 3, but peaked at week 14 [58].

\section{Physiological variability}

While we did not specifically research donorlevel characteristics that were likely to influence cytokine production, we did encounter a number of results that could be interpreted to influence donor variability. Some of these characteristics are unlikely to change dramatically during immunotherapy (genetic background, gender and age) while others are more likely to change during immunotherapy (stress and cachexia). These characteristics are discussed below.

\section{Gender}

One study reported higher spontaneous IFN- $\gamma$, higher cytomegalovirus (CMV)-specific IL-2 and lower influenza A-specific TNF- $\alpha$ production in CMV-seropositive women $(\mathrm{n}=29)$ than CMV-seropositive men $(n=21)$, as measured by ELISA in PBMC supernatants [59]. Another study presented gender-related patterns in urine cytokine levels in kidney transplant recipients with urinary tract infections [60]. Hahn et al. noted a significant difference in freedom from rejection of renal allografts between TNF- $\alpha$ high and TNF- $\alpha$ low genotype males, with no such difference for females [61]. The authors speculated that the hormones that predominate in females might have mitigating effects on TNF- $\alpha$ production, or that those that predominate in males may have exacerbating effects.

\section{Age}

Multiple studies discussed differences in cytokine production associated with donor age. Shurin et al. provided a comprehensive analysis of 30 cytokines in 397 healthy donors, ranging in age from 40 to 80 years [62]. Finnerty et al. studied longitudinal cytokine production in pediatric and adult burn patients, identifying multiple statistically significant differences between children and adults, and different kinetics between the two cohorts [13]. Bernstein et al. noted that $13 \%$ of elderly patients (approximately 80 years of age) did not produce detectable levels of IL-6, IL-10 or IFN- $\gamma$ before or after influenza vaccination [10].

\section{- Genetic background: HLA}

In two papers based on samples collected from 738 children who had been vaccinated for rubella, Ovsyannikova and colleagues discussed associations of cellular immune response with HLA class I and II alleles [47], and HLAbased haplotypes [63]. The authors measured rubella-specific T cells based on IFN- $\gamma$ and IL-10 ELISPOT, and the secretion of nine cytokines (IL-2, IL-4, IL-5, IL-6, IL-10, IL-12p70, IFN- $\gamma$, TNF- $\alpha$ and GM-CSF) in PBMC culture supernatants by ELISA. They found strong associations between multiple $H L A-D Q A 1$ and $H L A-D Q B 1$ alleles and IL-2, and suggestive relationships between $H L A-A, H L A-B$ and $H L A-C$ alleles and TNF- $\alpha$ [47]. Using inferred HLA-based haplotypes, they identified potential associations with IL-10 ELISPOT counts and IL-2, IL-10, TNF- $\alpha$ and IL-6 secretion [63]. In a study of five cytokines in systemic lupus erythematosus (SLE), Gómez and colleagues observed that cytokine monitoring results might be more informative if genetic background were taken into account, since other studies have shown that patients with the $H L A-D R^{*} 03, H L A-B^{*} 08$ 
and $H L A-A^{*} 01$ (3.8.1) haplotype tend to be high producers of TNF- $\alpha$ and IL-10 [64]. Taken together, these papers suggest that HLA genetic profiles and haplotypes may influence cytokine responses to immunotherapy. In addition, HLA was specifically mentioned by the MIATA project as being information that should be collected [55].

\section{- Genetic background: cytokine gene polymorphisms}

Multiple articles published in 2000 and 2001 examined the association of cytokine gene polymorphisms with post-transplantation outcomes. Allelic variations in TNF- $\alpha$, IFN- $\gamma$, TGF- $\beta 1$, IL-16 and IL-10 were studied in 56 pediatric liver transplant recipients [65]; TNF- $\alpha$, IFN- $\gamma$, IL-6, IL-10 and IL- 4 receptor- $\alpha$ in 120 renal transplant recipients [61]; and TNF- $\alpha$, IFN- $\gamma$, TGF- $\beta$ and IL-10 in 101 kidney or simultaneous kidney-pancreas recipients [66]. The TNF- $\alpha$ phenotype correlated with low TNF- $\alpha$ production was protective in both kidney and kidney-pancreas transplant recipients $[61,66]$, and was associated with successful withdrawal from immunosuppression in pediatric liver transplant recipients. Hahn et al. commented that the association between the TNF- $\alpha$ high genotype and graft outcome was not seen in recipients of a HLA-DR matched allograft. They argued that in this case, the recipient's helper $T$ cells are less likely to become stimulated to produce cytokines by proteins recognized as foreign, and thus the impact of the genetic difference is not manifested [61]. More recently, Lee and colleagues studied single nucleotide polymorphisms (SNPs) in eight cytokine and cytokine receptor genes (IL-1, IL-1R, IL-2, IL-4, IL-4R, IL-10, TGF- $\beta 1$ and IFN- $\gamma$ ) in 170 patients with colorectal cancer and 130 healthy controls. They reported that IL- $4 \mathrm{R}$ and TGF- $\beta 1$ polymorphisms are associated with the risk of colorectal cancer in a Korean population [67]. In a 2010 report of the immune responses of 738 children to rubella vaccines, Dhiman et al. identified SNPs and haplotypes in TNFa/TNFRSF1B and $I L 12 B$ genes that are associated with modulated immune responses to rubella vaccination [68]. Collectively, these studies suggest that cytokine gene polymorphisms might be one source of donor variability in cytokine production.

\section{Cachexia, stress \& sleep efficiency}

Cancer cachexia is weight loss, anorexia and loss of body cell mass in response to a malignant growth. One study measured TNF- $\alpha$,
IL- 6 and IL-1RA in cachectic cancer patients, concluding that increases in TNF- $\alpha$ and IL-6 might be useful markers for the evaluation of cachexia itself [69]. In addition, body weight was one of five clinical factors predictive of survival in a clinical trial of sipuleucel-T, an active cellular immunotherapy for cancer consisting of autologous dendritic cells that have been pulsed ex vivo with a fusion protein [70]. In a study of cytokine abnormalities in panic disorder and post-traumatic stress disorder, Hoge and colleagues reported that $87 \%$ of anxiety patients showed detectable levels of six of nine proinflammatory cytokines compared with only $25 \%$ of controls [44]. Another study noted that greater sleep efficiency was correlated with lower plasma levels of IL- 6 in 74 women aged 61-90 years [71]. Thus, one might speculate that donor-level characteristics such as weight loss, stress or sleep efficiency that might change during immunotherapy could have their own associated impacts on cytokine profiles.

\section{Data analysis \& presentation}

An appreciation of the repertoire of proven analytical techniques can aid in the interpretation of data from cytokine monitoring studies. The papers reviewed here presented numerical summaries, graphical representations and inherently multidimensional techniques encompassing both data mining and statistical approaches. A few of these techniques capture donor diversity. Such techniques help to accommodate the interdonor variability discussed in the above section. Importantly, the graphical technique used to display data can have a surprising impact on the interpretation of the results. In order to illustrate this, we provide some specific examples of graphical techniques and associated nuances. In addition, we catalog the techniques used in the literature summarized in Tables $1 \& 2$. This approach is intended to give readers a succinct overview of current practices in data analysis and presentation of cytokine profiles.

\section{Numerical summaries}

Some studies provided useful numerical summaries of their data, thereby documenting representative effect sizes, and potentially informing sample size calculations for future work. Gómez et al. showed means \pm SD for five cytokines, measured by ELISA in serum collected from 52 SLE patients and 25 controls. The numbers were also broken down by three different SLE Disease Activity Index (SLEDAI) ranges [64]. Hoge et al. provided means \pm SD and medians 
for 20 cytokines in 28 post-traumatic stress disorder patients and 28 age-matched controls, in addition to 20 panic disorder patients and 20 age-matched controls. Cytokines were measured in plasma by Luminex [44]. Takahashi et al. reported means \pm SD of 15 cytokines and growth factors in 122 patients with psoriasis and 78 healthy controls. Cytokines were measured in serum by ELISA [36]. Both Burgdorf and Dehqanzada presented the median and range of 15 and 22 cytokines, respectively [25,32]. Collectively, these studies provide valuable reference points for future work.

\section{Scatter plots, bar plots \& box plots}

To illustrate the differences between cohorts or treatments for a single cytokine, authors used scatter plots $[33,38]$, bar plots [25,72] and/or box plots [37,62]. Sets of multiple such plots show the results for multiple cytokines but do not address patterns of cytokine response at a donor level. The type of graph used to display data influences the interpretation of the results. To illustrate this, a representative scatter plot, bar plot and two box plots are shown in Figure 1. The data underlying the graphs is inspired by results presented by Inagaki et al. [38], although the data shown here is artificial. Scatter plots show one point for each sample, sometimes with random horizontal offset (jitter) to minimize overplotting. In this example, there are so many points near zero that overplotting still exists. Both bar plots and box plots represent each cohort with a single rectangle, coupled with additional annotations to show features of the population. In this case, the height of each bar is the mean of the samples in the cohort, while the error bars represent the $95 \%$ confidence interval for that mean. Bar plots can also be used to represent a single number, such as the response level of one donor. In the box plot, the box represents the interquartile range (25th percentile through 75 th percentile) with a heavy line at the median. In Figure 1, Box plot 1, the whiskers extend to the minimum and maximum values of the population. In Figure 1, Box рцот 2, the whiskers extend to 1.5-times the interquartile range, with the outlying points explicitly plotted. The NIST/SEMATECH e-Handbook of Statistical Methods provides additional information on plot types, appropriate applications and associated nuances [102].

\section{Visualizing stratification \& separation}

Given the diversity of human responses, data may suggest stratifying donors based on response patterns. In looking at responses for one variable at a time, scatter plots facilitate identification of stratification within a cohort and separation across cohorts. Bar plots obscure theses patterns,

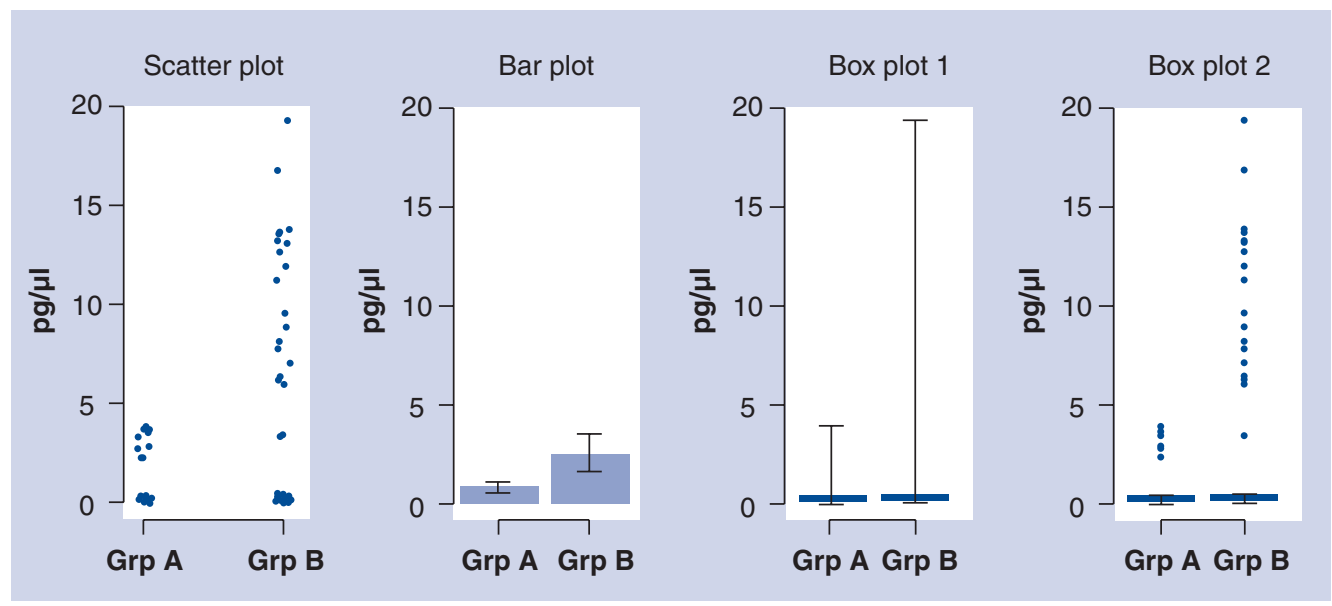

Figure 1. Graphic representations of the same data set. In the scatter plot, with one point per sample, we can see that both Group A and Group B show stratification, perhaps into nonresponders (the points in the large groups near 0 ) and responders. Additionally, the responders in Group B generally show a much higher response than the responders in Group A. Unfortunately, neither the stratification nor the separation are discernable in the bar plot. The separtion between Group A and Group B is suggested by box plot 2. Both bar plots and box plots represent each cohort with a single rectangle, coupled with additional annotations to show features of the population. In this case, the height of the bar plot is the mean of the samples, and the error bars represent the $95 \%$ confidence interval for that mean. In the box plot, the box represents the interquartile range (25th percentile through 75th percentile). In box plot 1, the whiskers extend to the minimum and maximum values of the population. In box plot 2, the whiskers extend to 1.5-times the interquartile range, with the outlying points explicitly plotted. 
as shown in Figure 1. In the scatter plot, we can see that both Group A and Group B show stratification, perhaps into nonresponders (the points in the large groups near 0) and responders. In addition, the responders in Group B generally show a much higher response than the responders in Group A. Unfortunately, these insights are not discernable in the bar plot or the box plots. Bar plots aggregating cohort data (as opposed to illustrating readouts at a donor level) were used in 14 of the 38 (37\%) publications summarized in Taвles $1 \& 2$, and were the only graphical technique used in six of the publications. Such aggregate representations obscure both donorlevel detail and potentially important patterns such as separation and stratification.

In studies in which only a fraction of the patients respond to treatment, analytical techniques that highlight intra-cohort stratification are essential, since these observations could lead to the ability to predict patients who are likely to respond to a therapy [73]. Once such stratification is identified, biobanked specimens from the original study could be examined in an effort to identify the underlying causes of response

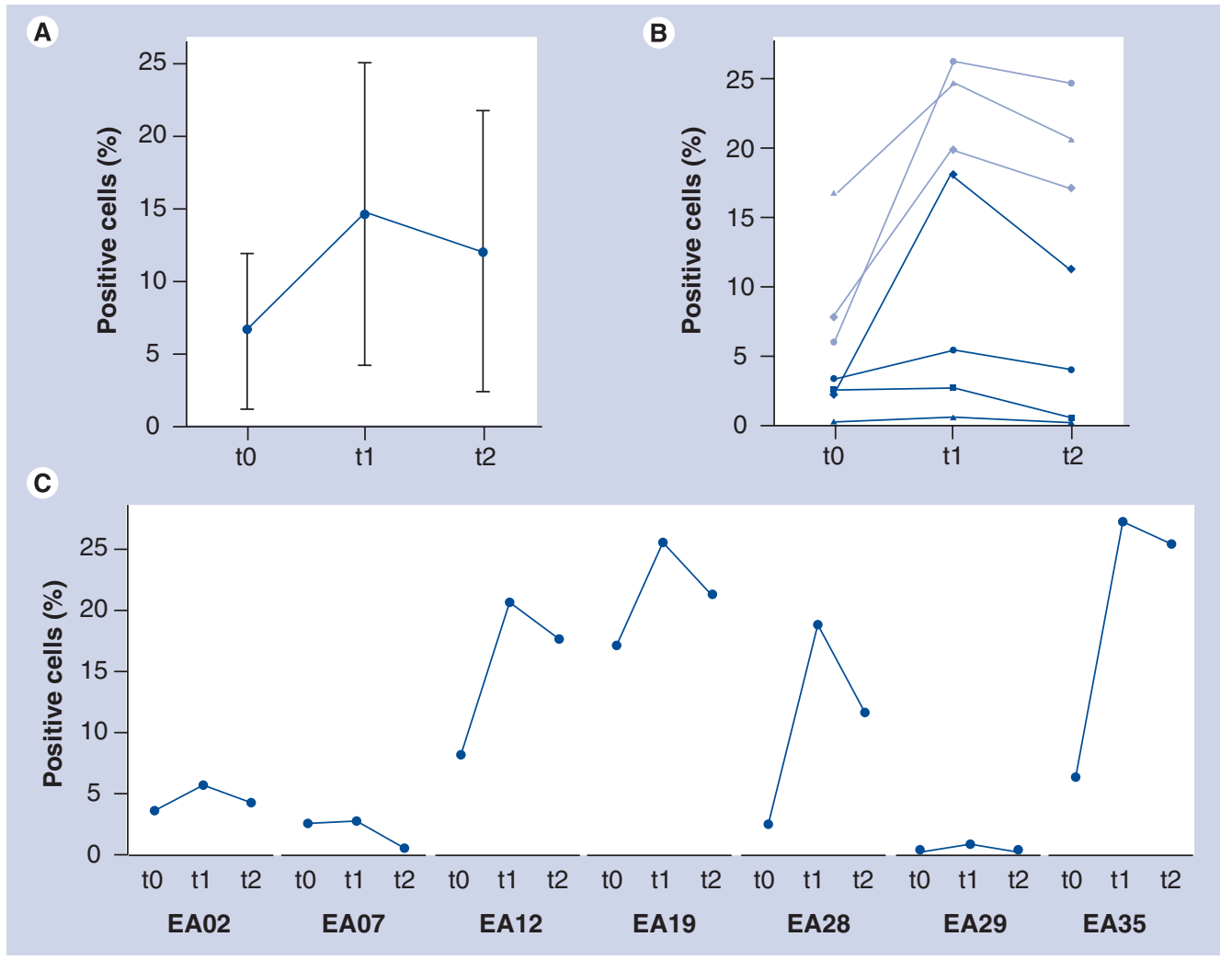

Figure 2. Three styles of longitudinal profiles, consisting of three time points for seven patients undergoing immunotherapy. (A) is an aggregate longitudinal profile showing the cross-donor mean at each timepoint, with error bars representing standard deviations. (B) is an overlay of individual donor profiles. (C) shows data for each donor (e.g., EA02) in its own subplot, which is an application of the 'small multiples' technique. The differences among donors that are clearly visible in the overlay and small multiples are not discernable in the aggregate profile. 
profile. However, neither the overlay nor the small multiples format can clearly represent differences among donors if there are hundreds of donors in the study. The following sections discuss applications of these three techniques.

\section{Aggregate profiles}

In the studies reviewed in Tables 1 \& 2, aggregate longitudinal profiles were used to illustrate data for upwards of five time points. Burgdorf et al. presented data for five cytokines monitored in 14 donors with colorectal cancer receiving a dendritic cell-based vaccine. Cytokines were measured at five time points in plasma using Luminex. Longitudinal profiles were shown for two cohorts, those patients with progressive disease and those with stable disease. Data was presented as the median of the cohort. Statistically significant differences, as computed by the Mann-Whitney $U$ test, were shown for GM-CSF, IFN- $\gamma$, IL-2, IL-5 and TNF- $\alpha$. In addition, numeric summaries of median and range across all patients and all time points were presented for 14 cytokines [32]. In a study of pediatric and adult burn patients $(n=24$ and $\mathrm{n}=25$, respectively), Finnerty et al. illustrated longitudinal profiles for 22 cytokines for six time points. Profiles were presented as cohort mean \pm standard error of the mean. Statistical differences were computed using Student's t-test. Data was collected from plasma samples using a CBA. Surprisingly, the authors removed outliers, defined as those observations outside the mean \pm SD, from their analysis [13]. Krause et al. also showed aggregate longitudinal profiles for 43 recipients of autologous peripheral blood stem cell transplants. Data for five cytokines, collected from plasma using ELISA, was presented as the median, and 15 th and 85 th percentiles across six time points. Reference data from ten healthy controls at a single time point was also shown. Significant changes from one time point to another were computed using a two-sided Mann-Whitney U test. In addition, 22 different leukocyte subsets, as measured by eight different flow cytometry panels, were monitored [14]. While these aggregate profiles illustrate longitudinal patterns for multiple cytokines, they mask donor-level diversity and the possible existence of meta-patterns common to multiple donors.

\section{Overlay profiles}

The inherent diversity in human populations is perhaps the most intriguing and most frustrating part of studying human disease and immune response. Several authors presented donor-level overlays showing changes of a particular readout for each and every donor. Christensen $e t a l$. presented such plots for nine cytokines expressed pre- and post-treatment in the serum of 42 prostate cancer patients undergoing intensitymodulated radiotherapy. Significant differences were calculated using the Mann-Whitney $U$ test [40]. Takahashi et al. illustrated pre- and post-treatment serum TNF- $\alpha$ and IL-10 levels

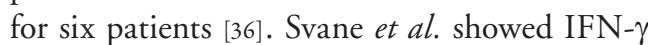
as measured by ELISA, for up four time points. Data was collected from PBMCs of breast cancer patients vaccinated with $\mathrm{p} 53$ peptide-pulsed dendritic cells [15]. Such overlays provide insight into donor-level longitudinal patterns, and are legible for a small number of donors. Unfortunately, plots with 42 donors are difficult to interpret due to overplotting.

\section{Representative donor profiles}

Hrouda et al. illustrated donor-level longitudinal profiles of the percentage of cells expressing intracellular cytokine production of IFN- $\gamma$, IL- 2 or IL- 4 as measured by flow cytometry in prostate cancer patients treated with heat-killed Mycobacterium vaccae. Data was shown for up to 17 time points [2]. Tan $e t$ al. provided particularly elegant donor-level profiles integrating viral load, serum alanine aminotransferase (ALT) and IL-10 across eight time points. In addition, phases of disease and treatment (under treatment, viral rebound and hepatic flare), as defined by increasing or decreasing levels of viral load and ALT, were captured in the profile. As such, these graphs offered a 'composite array of immunological parameters' [43]. In both of these studies, longitudinal profiles of representative donors were shown, in contrast to small multiple representations of all donors. While the physical space required to render profiles for all donors for all time points for all cytokines generally exceeds the space available in publications, inspection of such profiles can be a valuable step in initial exploration of the data. Thus, the techniques that are useful for prepublication data analysis may be different from the techniques that are used in a formal written presentation

\section{Polyfunctionality}

The concept of polyfunctionality has been applied in a number of studies using multiparameter flow cytometry to reduce and summarize the data at a sample level $[27,34,35,48,77,78]$. Essentially, multiple cytokines or other functional parameters are measured, and readouts are presented as the percentage of cells producing, 
for example, 5, 4, 3, 2 or 1 of the parameters. Commonly, CD107a, IFN- $\gamma$, IL-2, MIP1 $\beta$ and TNF- $\alpha$ are measured $[27,34,77]$. Multiple studies demonstrated that HIV-specific CD8 ${ }^{+} \mathrm{T}$ cells of HIV nonprogressors tend to produce multiple analytes $[34,77]$. Other studies suggested that polyfunctionality is a correlate of vaccinemediated protection [27,35], while Yuan et al. showed that a CTLA-4 blockade increased the polyfunctionality of NY-ESO-1-specific $T$ cells [48]. Often this polyfunctionality data was illustrated using the software SPICE [103]. Illustrations captured data for either a single sample, or the mean of multiple samples. These polyfunctionality studies offer an additional line of evidence on the value of measuring multiple cytokines simultaneously, since polyfunctionality has been shown to correlate with clinical outcome or prognosis.

\section{- Donor-level data normalization}

One approach that works well for mitigating donor-level diversity while accommodating multiple readouts is the creation of donor-level profiles using normalized data $[79,80]$. Essentially, for each donor, for each aliquot (e.g., time point and ex vivo stimulation condition), all readouts can be mapped onto a scale from 0 to 1 , with the highest readout being mapped to 1 and the lowest to 0 . The resulting data can be analyzed with standard statistical techniques regardless of the original magnitude of the readouts.

\section{Multidimensional techniques}

Studies that measure more than two or three cytokines and more than two or three clinical parameters across multiple patient cohorts generally require multidimensional/multivariate approaches to fully interrogate the data. Techniques commonly used with microarray data, such as heatmaps and clustering, can be applied to cytokine data. A variety of general machine learning and data mining techniques and statistical models are also applicable. In addition, multiple techniques can be chained together in an analytical workflow or pipeline $[45,80,81]$. Software tools leveraged in the articles reviewed here include $\mathrm{R}$ [82], Matlab [81], Weka [81] and TIGR MEV [83]. R, TIGR MEV and Weka are freely available online.

\section{Microarray style techniques}

In a study of cytokine secretion patterns of human PBMCs in response to stimulation with Toll-like receptors (TLRs), Kattah et al. applied an interesting data analysis approach [83]. First, to filter the readouts for 22 cytokines, they used a standard microarray analysis algorithm, 2-class Significance Analysis of Microarrays, which is available in the TIGR MEV program [84]. This allowed them to identify six cytokines that were differentially expressed in response to TLR stimulation. Of these six, they focused on five that were present at reasonably high levels. Then, using all possible combinations of these five cytokines (32 total), they identified the combinations that led to IL-17 production. In a different application of microarray style techniques, de Jager $e t$ al. and van den Ham et al. used heat maps and cluster analysis to illustrate differential cytokine profiles in osteoarthritis and rheumatoid arthritis [16] and in juvenile idiopathic arthritis [82]. Thus, techniques popularized to address the challenges of microarray data analysis can be applied to cytokine profile data.

\section{Data mining techniques}

Cruz and Wishart reviewed the application of machine learning techniques such as artificial neural networks (ANN) and support vector machines (SVM) to cancer prediction and prognosis [85]. In a study of 26 prostate cancer patients vaccinated with irradiated allogenic cells, Michael et al. employed multiple immune monitoring assays including: cell surface phenotypes as measured by flow cytometry; proliferation in response to tumor lysate, as measured by bromodeoxyuridine (BrdUrd); nonspecific cytokine release in response to stimuli including phorbol myristate acetate, lipopolysaccharides and concanavalin A; spontaneous cytokine release as measured with a CBA; and expression of cytokines as measured by real-time PCR. To analyze the resulting complex multiparameter data set, they employed an ANN that was able to identify responders with $84 \%$ accuracy on test data. Interestingly, the optimal set of input variables to the ANN included parameters measured by PCR, BrdUrd proliferation, cell surface phenotype, and the CBA [86]. McKinney and colleagues used a combination of statistical techniques and machine learning techniques (SVM, nearest shrunken centroid and decision tree) to predict adverse events following smallpox immunization. Input into their models was serum expression levels of 108 protein analytes as measured by a custom protein array [81]. Thus, there are a variety of data mining techniques that can be applied to cytokine profiles. However, as a whole, one weakness of these techniques is that biological interpretation of the numerical results may be difficult. 


\section{Statistical models}

The work presented by Casasnovas et al. [45] and Inagaki et al. [38] both apply classical statistical techniques to identify prognostic factors for Hodgkin's lymphoma and adult $\mathrm{T}$ cell leukemia/lymphoma (ATLL), respectively. The first study measured six cytokines and soluble receptors in plasma of 519 patients and 32 healthy controls recruited from 17 centers over a 4 year period. The second study measured eight cytokines in the serum of 94 ATLL patients and 50 healthy controls. Patients were diagnosed over a 16 year period at one of two hospitals. Both studies used Kaplan-Meir survival analysis and Cox proportional hazard models. Both studies also established analyte-specific cutoff values to stratify patients in 'high' or 'low' groups, with patients having one classification for each analyte.

From this type of analysis using standard statistical models, Casasnovas et al. derived a 3-marker prognostic index based on soluble CD30, IL-6 and IL-1RA, and stratified patients based on zero to three positive markers [45]. 5-year overall survival for patients with no positive markers was $95 \%$, dropping to $63 \%$ for those patients with three positive markers. This approach is similar to that used in diabetes autoantibody monitoring $[7,8]$. Hoge et al. also identified a multi-marker signature (detectable levels of six of nine proinflammatory cytokines) that was present in $87 \%$ of anxiety patients but only $25 \%$ of controls [44]. In summary, standard statistical models can support the identification of multi-marker signatures associated with clinical outcome.

\section{Thinking multidimensionally}

Taken together, this literature illustrates the application of multidimensional analytical techniques to cytokine monitoring and immunotherapy. One important aspect of these techniques is that, for the most part, they are extensible to tens or even hundreds of readouts, with acceptable computational performance. Thus they can readily be applied to data measuring six or 60 cytokines. However, neither reviews nor research articles are written with the intent of providing a thorough background on the algorithm, nuances and general applicability of a particular technique. The successful application of a published approach to a new data set requires the willingness to thoroughly understand the technique and/or collaboration with appropriate experts. Likewise, experts in analytical approaches are better able to design analytical strategies if they are conversant in the biology associated with the data.
Additionally, both statistical and data mining approaches may generate results that are numerically correct, but biologically uninterpretable or unbelievable. A straightforward example of this phenomenon is when a simple statistical test such as Student's t-test shows a significant difference between two populations, but the numerical difference between the means of the populations is too small to be considered biologically relevant. Therefore, the multidimensional techniques discussed here are best described as 'potentially useful.' On the other hand, traditional univariate techniques alone are often unsatisfying for this multivariate data.

In summary, to successfully analyze and interpret the results of large-scale multi-time point, multi-analyte and potentially multi-platform monitoring studies, we must be prepared to apply and compare a variety of analytical techniques. In this way, over time, we can build our repertoire of proven techniques. Furthermore, given the significant biological diversity that exists in human donors, we need to specifically seek out and develop more approaches that allow each donor to be his or her own control.

\section{Conclusion \& future perspective}

The cytokine response to immune stimulation is complex and multifaceted. Different cytokines have different roles and kinetics depending on the microenvironment. Furthermore, the physiological diversity of human populations adds to the complexity of monitoring cytokine profiles in response to immunotherapy. However, the body of literature reviewed here provides a solid foundation for designing increasingly sophisticated studies. The literature on assay optimization and standardization provides both valuable insights for controlling variability and helpful guidance for researchers wanting to optimize and standardize assays for their projects. In addition, the use of assay performance controls helps to characterize assay reproducibility and precision.

The studies using multiplex assays document the value of such assays and the numerical ranges of response under a variety of conditions. Future studies might combine multiplex assays with other procedures to create highly informative multipart assays for more definitive assessments of immune response. For example, since multiplex assays support the simultaneous analysis of 10-50 analytes, such assays can be used to screen samples for differentially expressed cytokines. Further analysis to isolate the cytokine signal to particular cell subsets can be accomplished through a combination of 
magnetic bead separation [87] and ICS combined with multiparameter cell surface phenotyping. Another approach is the initial separation of rare cell populations using polychromatic (5 to 15 color) flow cytometry. Once isolated, these populations can be subjected to ex vivo stimulation, with the resulting supernatant interrogated with a CBA.

Computational tools and techniques popularized and made readily available to address the challenges of microarray data offer a starting point for rich multidimensional analysis. In addition, data mining and multivariate statistical techniques may provide valuable insight. The literature reviewed here provides representative examples of applications of such tools. That said, we need additional analytical techniques that support the gleaning of meaningful biological insights from complex cytokine data sets, that help us to think multidimensionally, and that allow us to treat each donor as his or her own control. We also need more longitudinal studies of healthy controls so that we have a better sense of normal changes over time. In conclusion, with appropriate attention paid to details of assay optimization, donor-level diversity and multidimensional data analysis, monitoring cytokine profiles during immunotherapy offers great potential for innovation and discovery.

\footnotetext{
Financial \& competing interests disclosure

Janet Siebert is President and Founder of CytoAnalytics. The authors have no other relevant affliations or financial involvement with any organization or entity with a financial interest in or financial conflict with the subject matter or materials discussed in the manuscript apart from those disclosed.

No writing assistance was utilized in the production of this manuscript.
}

\section{Executive summary}

\section{Overview of monitoring}

- The existing literature inspecting cytokine profiles can be divided into 'measurement' studies and 'monitoring' studies. Measurement implies that observations are taken at a single time point, while monitoring implies at least two time points.

- Long-term large-scale monitoring studies in autoimmunity provide insight into what is possible.

- Large studies of immune response associated with infectious disease vaccinations provide a sense of range of responses.

Cytokine milieus

- While convenient, the Th1/Th2 distinction oversimplifies the complex nature of cytokine signaling.

\section{Platforms for monitoring cytokine profiles}

- Platforms for monitoring cytokine profiles include ELISA, ELISPOT, multiplex assays such as Luminex or cytokine bead arrays, intracellular cytokine flow cytometry and gene expression platforms.

- Multiplex assays enable the measurement of 10-50 cytokines and chemokines.

\section{Experimental design}

- Control constructs include comparing donors with disease to healthy controls, donors with one disease to donors with another disease, pretreatment readouts to post-treatment readouts, and using the individual donor as his or her own control.

- Reported readouts include: original assay data, such as picogram/milliliter; the mean or median of replicate measurements; backgroundsubtracted values such as stimulated readout minus a comparable unstimulated readout; or a combination thereof.

- Positive responses have been defined as post-treatment value exceeding pretreatment value, post-treatment greater than two or three times the pretreatment value, or the mean plus three standard deviations of the readout in a control group.

\section{Assay variability}

- A valuable body of literature addresses assay optimization, validation and standardization.

- Additional factors that effect assay variability are mentioned in some studies. Such factors include diurnal effects, absolute cell count and kinetics.

\section{Physiological variability}

- Donor-level characteristics likely to remain relatively unchanged during therapy include gender, age and genetic background.

- Donor-level characteristics that might change during therapy include cachexia, stress and sleep efficiency.

\section{Data analysis \& presentation}

- In general, data presentation consists of standard population-level numeric summaries such as mean, range and standard deviation; or standard graphical techniques such as scatter plots and box plots.

- Some studies present longitudinal profiles or time series graphs, either at a summarized level or at a donor level. The donor-level profiles are particularly useful as they allow each donor to serve as his or her own control.

- Multidimensional analytical techniques, such as those developed specifically to analyze microarray data or general-purpose data mining techniques, are occasionally used.

\section{Conclusion \& future perspective}

- The existing literature provides a solid basis for future studies.

- Multiplex assays, coupled with increasing availability of multidimensional analytical tools, facilitate the study of a complex cytokine response to immune stimulation.

- We need to improve our tools and skills for thinking multidimensionally. 


\section{Bibliography}

Papers of special note have been highlighted as:

- of interest

" of considerable interest

1 Clay TM, Hobeika AC, Mosca PJ, Lyerly HK, Morse MA: Assays for monitoring cellular immune responses to active immunotherapy of cancer. Clin. Cancer Res. 7, 1127-1135 (2001).

2 Hrouda D, Baban B, Dunsmuir WD, Kirby RS, Dalgleish AG: Immunotherapy of advanced prostate cancer: a Phase I/II trial using Mycobacterium vaccae (SRL172). Br. J. Urol. 82, 568-573 (1998).

3 Beachy SH, Repasky EA: Using extracellular biomarkers for monitoring efficacy of therapeutics in cancer patients: an update. Cancer Immunol. Immunother. 57, 759-775 (2008).

4 Kim H, Jin H, Lee S et al:: The effect of rush immunotherapy with house dust mite in the production of IL- 5 and IFN- $\gamma$ from the peripheral blood $\mathrm{T}$ cells of asthmatic children. J. Korean Med. Sci. 24, 392-397 (2009).

5 Minelli M, Schiavino D, Musca F et al:: Oral hyposensitization to nickel induces clinical improvement and a decrease in TH1 and TH2 cytokines in patients with systemic nickel allergy syndrome. Int. J. Immunopathol. Pharmacol. 23, 193-201 (2010).

6 Nieminen K, Laaksonen K, Savolainen J: Three-year follow-up study of allergeninduced in vitro cytokine and signalling lymphocytic activation molecule mRNA responses in peripheral blood mononuclear cells of allergic rhinitis patients undergoing specific immunotherapy. Int. Arch. Allergy Immunol. 150, 370-376 (2009).

7 Barker JM, Barriga KJ, Yu L et al.: Prediction of autoantibody positivity and progression to type 1 diabetes: Diabetes Autoimmunity Study in the Young (DAISY). J. Clin. Endocrinol. Metab. 89, 3896-3902 (2004).

8 Verge CF, Gianani R, Kawasaki E et al.: Number of autoantibodies (against insulin, GAD or ICA512/IA2) rather than particular autoantibody specificities determines risk of type I diabetes. J. Autoimmun. 9, 379-383 (1996).

9 Dhiman N, Haralambieva IH, Vierkant RA et al:: Predominant inflammatory cytokine secretion pattern in response to two doses of live rubella vaccine in healthy vaccinees. Cytokine 50, 24-29 (2010).

10 Bernstein ED, Gardner EM, Abrutyn E, Gross P, Murasko DM: Cytokine production after influenza vaccination in a healthy elderly population. Vaccine 16, 1722-1731 (1998).
11 Hoos A, Parmiani G, Hege K et al.: A clinical development paradigm for cancer vaccines and related biologics. J. Immunother. 30, 1-15 (2007).

12 Millán O, Urtasun N, Brunet M: Biomarkers of the immunomodulatory effect of immunosuppressive drugs in transplant recipients. Transplant Rev. (Orlando) 23, 120-128 (2009).

13 Finnerty CC, Jeschke MG, Herndon DN et al.: Temporal cytokine profiles in severely burned patients: a comparison of adults and children. Mol. Med. 14, 553-560 (2008).

- $\quad$ Aggregate longitudinal profiles of 22 cytokines across six time points in adult $(\mathrm{n}=25)$ and pediatric $(\mathrm{n}=24)$ severely burned patients.

14 Krause SW, Rothe G, Gnad M, Reichle A, Andreesen R: Blood leukocyte subsets and cytokine profile after autologous peripheral blood stem cell transplantation. Ann. Hematol. 82, 628-636 (2003).

- Detailed aggregate longitudinal profiles for five cytokines and multiple cell subsets before, during and after two rounds of autologous peripheral blood stem cell transplantation in 43 patients.

15 Svane IM, Pedersen AE, Johansen JS et al.: Vaccination with $\mathrm{p} 53$ peptide-pulsed dendritic cells is associated with disease stabilization in patients with $\mathrm{p} 53$ expressing advanced breast cancer; monitoring of serum YKL-40 and IL-6 as response biomarkers. Cancer Immunol. Immunother. 56, 1485-1499 (2007).

16 de Jager W, Bourcier K, Rijkers GT, Prakken $\mathrm{BJ}$, Seyfert-Margolis V: Prerequisites for cytokine measurements in clinical trials with multiplex immunoassays. BMC Immunol. 10 , 52 (2009).

17 van den Engel NK, Winter H, Rüttinger D et al:: Characterization of immune responses in gastric cancer patients: a possible impact of H. pylori to polarize a tumor-specific type 1 response? Clin. Immunol. 120, 285-296 (2006).

18 Kyte JA, Trachsel S, Risberg B et al.: Unconventional cytokine profiles and development of $\mathrm{T}$ cell memory in long-term survivors after cancer vaccination. Cancer Immunol. Immunother. 58, 1609-1626 (2009).

19 Suntharalingam G, Perry MR, Ward S et al.: Cytokine storm in a Phase 1 trial of the anti-CD28 monoclonal antibody TGN1412. N. Engl. J. Med. 355, 1018-1028 (2006).

20 Singh M, Basu S, Camell C et al.: Selective expansion of memory CD4(+) T cells by mitogenic human CD28 generates inflammatory cytokines and regulatory T cells. Eur. J. Immunol. 38, 1522-1532 (2008).
21 van der Vliet HJJ, Molling JW, Nishi N et al.: Polarization of $\mathrm{V} \alpha-24^{+} \mathrm{V} \beta-11^{+}$natural killer $T$ cells of healthy volunteers and cancer patients using $\alpha$-galactosylceramide-loaded and environmentally instructed dendritic cells. Cancer Res. 63, 4101-4106 (2003).

22 Veldhoen M, Seddon B: Empowering T helper 17 cells in autoimmunity. Nat. Med. 16, 166-168 (2010).

23 Veldhoen $\mathrm{M}$ : The role of $\mathrm{T}$ helper subsets in autoimmunity and allergy. Curr. Opin. Immunol. 21, 606-611 (2009).

24 Kijanka G, Murphy D: Protein arrays as tools for serum autoantibody marker discovery in cancer. J. Proteomics 72, 936-944 (2009).

25 Dehqanzada ZA, Storrer CE, Hueman MT et al.: Assessing serum cytokine profiles in breast cancer patients receiving a HER2/neu vaccine using Luminex technology. Oncol. Rep. 17, 687-694 (2007).

26 Quast S, Zhang W, Shive C et al:: IL-2 absorption affects IFN- $\gamma$ and IL-5, but not IL-4 producing memory $\mathrm{T}$ cells in double color cytokine ELISPOT assays. Cell. Immunol. 237, 28-36 (2005).

27 Precopio ML, Betts MR, Parrino J et al.: Immunization with vaccinia virus induces polyfunctional and phenotypically distinctive CD8(+) T cell responses. J. Exp. Med. 204, 1405-1416 (2007).

28 Soares AP, Scriba TJ, Joseph S et al.: Bacillus Calmette-Guérin vaccination of human newborns induces $\mathrm{T}$ cells with complex cytokine and phenotypic profiles. J. Immunol. 180, 3569-3577 (2008).

29 Karlsson AC, Martin JN, Younger SR et al.: Comparison of the ELISPOT and cytokine flow cytometry assays for the enumeration of antigen-specific T cells. J. Immunol. Methods 283, 141-153 (2003).

30 Whiteside TL, Zhao Y, Tsukishiro T et al.: Enzyme-linked immunospot, cytokine flow cytometry, and tetramers in the detection of T-cell responses to a dendritic cell-based multipeptide vaccine in patients with melanoma. Clin. Cancer Res. 9, 641-649 (2003).

31 Liu Z, Yuan X, Luo Y et al.: Evaluating the effects of immunosuppressants on human immunity using cytokine profiles of whole blood. Cytokine 45, 141-147 (2009).

32 Burgdorf SK, Claesson MH, Nielsen HJ, Rosenberg J: Changes in cytokine and biomarker blood levels in patients with colorectal cancer during dendritic cell-based vaccination. Acta Oncol. 48, 1157-1164 (2009).

33 McElhaney JE, Xie D, Hager WD et al.: $\mathrm{T}$ cell responses are better correlates of vaccine protection in the elderly. J. Immunol. 176, 6333-6339 (2006). 
34 Betts MR, Nason MC, West SM et al.: HIV nonprogressors preferentially maintain highly functional HIV-specific CD8 ${ }^{+} \mathrm{T}$ cells. Blood 107, 4781-4789 (2006).

35 Darrah PA, Patel DT, De Luca PM et al: : Multifunctional TH1 cells define a correlate of vaccine-mediated protection against Leishmania major. Nat. Med. 13, 843-850 (2007).

36 Takahashi H, Tsuji H, Hashimoto Y, Ishida-Yamamoto A, Iizuka H: Serum cytokines and growth factor levels in Japanese patients with psoriasis. Clin. Exp. Dermatol. 35(6), 645-649 (2009).

37 Dufour C, Ferretti E, Bagnasco F et al.: Changes in cytokine profile pre- and post-immunosuppression in acquired aplastic anemia. Haematologica 94, 1743-1747 (2009).

38 Inagaki $\mathrm{A}$, Ishida $\mathrm{T}$, Ishii $\mathrm{T}$ et al: : Clinical significance of serum Th1-, Th2- and regulatory $\mathrm{T}$ cells-associated cytokines in adult T-cell leukemia/lymphoma: high interleukin-5 and -10 levels are significant unfavorable prognostic factors. Int. J. Cancer 118, 3054-3061 (2006).

39 Kimura A, Sakurai T, Koumura A et al.: Longitudinal analysis of cytokines and chemokines in the cerebrospinal fluid of a patient with Neuro-Sweet disease presenting with recurrent encephalomeningitis. Intern. Med. 47, 135-141 (2008).

40 Christensen E, Pintilie M, Evans KR et al.: Longitudinal cytokine expression during IMRT for prostate cancer and acute treatment toxicity. Clin. Cancer Res. 15, 5576-5583 (2009).

41 Khatri VP, Fehniger TA, Baiocchi RA et al.: Ultra low dose interleukin-2 therapy promotes a type 1 cytokine profile in vivo in patients with AIDS and AIDS-associated malignancies. J. Clin. Invest. 101, 1373-1378 (1998).

42 Zhang W, Liu S, Cao X et al.: A Phase-I clinical trial of active immunotherapy for acute leukemia using inactivated autologous leukemia cells mixed with IL-2, GM-CSF, and IL-6. Leuk. Res. 29, 3-9 (2005).

43 Tan AT, Koh S, Goh W et al.: A longitudinal analysis of innate and adaptive immune profile during hepatic flares in chronic hepatitis B. J. Hepatol. 52, 330-339 (2010).

- Elegant graphical representation of individual donor disease state, clinical readouts and cytokine production.

44 Hoge EA, Brandstetter K, Moshier S et al.: Broad spectrum of cytokine abnormalities in panic disorder and posttraumatic stress disorder. Depress. Anxiety 26, 447-455 (2009).
- Numerical summaries for 20 cytokines measured by Luminex in two patient cohorts, compared with age and gender-matched controls.

45 Casasnovas R, Mounier N, Brice P et al.: Plasma cytokine and soluble receptor signature predicts outcome of patients with classical Hodgkin's lymphoma: a study from the Groupe d'Etude des Lymphomes de l'Adulte. J. Clin. Oncol. 25, 1732-1740 (2007).

- Large prospective multicenter study of 519 patients resulting in three marker prognostic index for Hodgkin's lymphoma.

46 Larsen CE, Xu J, Lee S et al:: Complex cytokine responses to hepatitis B surface antigen and tetanus toxoid in responders, nonresponders and subjects naive to hepatitis B surface antigen. Vaccine 18, 3021-3030 (2000).

47 Ovsyannikova IG, Ryan JE, Vierkant RA et al.: Influence of host genetic variation on rubella-specific $T$ cell cytokine responses following rubella vaccination. Vaccine 27 , 3359-3366 (2009).

48 Yuan J, Gnjatic S, Li H et al.: CTLA-4 blockade enhances polyfunctional NY-ESO-1 specific $T$ cell responses in metastatic melanoma patients with clinical benefit. Proc. Natl Acad. Sci. USA 105, 20410-20415 (2008).

49 Horton H, Thomas EP, Stucky JA et al.: Optimization and validation of an 8-color intracellular cytokine staining (ICS) assay to quantify antigen-specific $\mathrm{T}$ cells induced by vaccination. J. Immunol. Methods 323, 39-54 (2007).

- Informative discussion of validation of an ICS assay in accordance with FDA guidance on Bioanalytical Method Validation.

50 Comin-Anduix B, Gualberto A, Glaspy JA et al.: Definition of an immunologic response using the major histocompatibility complex tetramer and enzyme-linked immunospot assays. Clin. Cancer Res. 12, 107-116 (2006).

51 Nomura L, Maino VC, Maecker HT: Standardization and optimization of multiparameter intracellular cytokine staining. Cytometry A 73, 984-991 (2008).

52 Maecker HT, Rinfret A, D'Souza P et al.: Standardization of cytokine flow cytometry assays. BMC Immunol. 6, 13 (2005).

53 Britten CM, Gouttefangeas C, Welters MJP et al:: The CIMT-monitoring panel: a two-step approach to harmonize the enumeration of antigen-specific $\mathrm{CD} 8^{+}$ T lymphocytes by structural and functional assays. Cancer Immunol. Immunother. 57, 289-302 (2008).
54 Janetzki S, Panageas KS, Ben-Porat L et al.: Results and harmonization guidelines from two large-scale international Elispot proficiency panels conducted by the Cancer Vaccine Consortium (CVC/SVI). Cancer Immunol. Immunother. 57, 303-315 (2008).

55 Janetzki S, Britten CM, Kalos M et al.: 'MIATA'-minimal information about $\mathrm{T}$ cell assays. Immunity 31, 527-528 (2009).

56 Hoffmeister B, Bunde T, Rudawsky IM, Volk $\mathrm{H}$, Kern F: Detection of antigen-specific $\mathrm{T}$ cells by cytokine flow cytometry: the use of whole blood may underestimate frequencies. Eur. J. Immunol. 33, 3484-3492 (2003).

57 Butterfield LH, Disis ML, Fox BA et al.: A systematic approach to biomarker discovery; preamble to 'the iSBTc-FDA taskforce on immunotherapy biomarkers'. J. Transl. Med. 6, 81 (2008).

- Highlights a number of issues relevant to discovery and validation of immunotherapy biomarkers.

58 Carbone DP, Ciernik IF, Kelley MJ et al.: Immunization with mutant $\mathrm{p} 53$ - and $\mathrm{K}$-ras-derived peptides in cancer patients: immune response and clinical outcome. J. Clin. Oncol. 23, 5099-5107 (2005).

59 Villacres MC, Longmate J, Auge C, Diamond DJ: Predominant type 1 CMV-specific memory T-helper response in humans: evidence for gender differences in cytokine secretion. Hum. Immunol. 65, 476-485 (2004).

60 Sadeghi M, Daniel V, Naujokat C et al: Strong inflammatory cytokine response in male and strong anti-inflammatory response in female kidney transplant recipients with urinary tract infection. Transpl. Int. 18, 177-185 (2005).

61 Hahn AB, Kasten-Jolly JC, Constantino DM et al: : TNF- $\alpha$, IL-6, IFN- $\gamma$, and IL-10 gene expression polymorphisms and the IL-4 receptor $\alpha$-chain variant Q576R: effects on renal allograft outcome. Transplantation 72, 660-665 (2001).

62 Shurin GV, Yurkovetsky ZR, Chatta GS et al.: Dynamic alteration of soluble serum biomarkers in healthy aging. Cytokine 39, 123-129 (2007).

63 Ovsyannikova IG, Vierkant RA, Pankratz VS et al:: HLA haplotype and supertype associations with cellular immune responses and cytokine production in healthy children after rubella vaccine. Vaccine 27, 3349-3358 (2009).

64 Gómez D, Correa PA, Gómez LM et al.: Th1/Th2 cytokines in patients with systemic lupus erythematosus: is tumor necrosis factor $\alpha$ protective? Semin. Arthritis Rheum. 33, 404-413 (2004). 
65 Mazariegos GV, Reyes J, Webber SA et al.: Cytokine gene polymorphisms in children successfully withdrawn from immunosuppression after liver transplantation. Transplantation 73 , 1342-1345 (2002).

66 Pelletier R, Pravica V, Perrey C et al.: Evidence for a genetic predisposition towards acute rejection after kidney and simultaneous kidney-pancreas transplantation. Transplantation 70, 674-680 (2000).

67 Lee Y, Choi H, Lee I, Kim T, Oh S: Association between IL-4R and TGF- $\beta 1$ gene polymorphisms and the risk of colorectal cancer in a korean population. Colorectal Dis. DOI: $10.1111 / j .1463-1318.2009 .02080 . x$ (2009) (Epub ahead of print).

68 Dhiman N, Haralambieva IH, Kennedy RB et al.: SNP/haplotype associations in cytokine and cytokine receptor genes and immunity to rubella vaccine. Immunogenetics $62,197-210$ (2010).

69 Takahashi M, Terashima M, Takagane A et al.: Ghrelin and leptin levels in cachectic patients with cancer of the digestive organs. Int. J. Clin. Oncol. 14, 315-320 (2009).

70 Small EJ, Schellhammer PF, Higano CS et al.: Placebo-controlled Phase III trial of immunologic therapy with sipuleucel-T (APC8015) in patients with metastatic, asymptomatic hormone refractory prostate cancer. J. Clin. Oncol. 24, 3089-3094 (2006).

71 Friedman EM, Hayney MS, Love GD et al: Social relationships, sleep quality, and interleukin-6 in aging women. Proc. Natl Acad. Sci. USA 102, 18757-18762 (2005).

72 Jiménez R, Ramírez R, Carracedo J et al.: Cytometric bead array (CBA) for the measurement of cytokines in urine and plasma of patients undergoing renal rejection. Cytokine 32, 45-50 (2005).

73 Copier J, Whelan M, Dalgleish A: Biomarkers for the development of cancer vaccines: current status. Mol. Diagn. Ther. 10, 337-343 (2006).

74 Kalos M: An integrative paradigm to impart quality to correlative science. J. Transl. Med. 8, 26 (2010).

75 Walker EB, Haley D, Petrausch U et al.: Phenotype and functional characterization of long-term gp100-specific memory CD8 ${ }^{+}$ $\mathrm{T}$ cells in disease-free melanoma patients before and after boosting immunization. Clin. Cancer Res. 14, 5270-5283 (2008).

76 Tufte ER: Visual Explanations. Graphic Press LLC, Cheshire, CT, USA (1997).

77 Streeck H, Brumme ZL, Anastario M et al.: Antigen load and viral sequence diversification determine the functional profile of HIV-1-specific CD8+ T cells. PLoS Med. 5, e100 (2008).
78 Casazza JP, Betts MR, Price DA et al.: Acquisition of direct antiviral effector functions by $\mathrm{CMV}$-specific $\mathrm{CD} 4^{+} \mathrm{T}$ lymphocytes with cellular maturation. J. Exp. Med. 203, 2865-2877 (2006).

79 Inokuma M, dela Rosa C, Schmitt C et al.: Functional $\mathrm{T}$ cell responses to tumor antigens in breast cancer patients have a distinct phenotype and cytokine signature. $J$. Immunol. 179, 2627-2633 (2007).

80 Siebert JC, Inokuma M, Waid DM et al: : An analytical workflow for investigating cytokine profiles. Cytometry A 73, 289-298 (2008).

81 McKinney BA, Reif DM, Rock MT et al.: Cytokine expression patterns associated with systemic adverse events following smallpox immunization. J. Infect. Dis. 194, 444-453 (2006).

82 van den Ham H, de Jager W, Bijlsma JWJ, Prakken BJ, de Boer RJ: Differential cytokine profiles in juvenile idiopathic arthritis subtypes revealed by cluster analysis. Rheumatology 48, 899-905 (2009).

83 Kattah MG, Wong MT, Yocum MD, Utz PJ: Cytokines secreted in response to Toll-like receptor ligand stimulation modulate differentiation of human Th17 cells. Arthritis Rheum. 58, 1619-1629 (2008).

84 Saeed AI, Bhagabati NK, Braisted JC et al: TM4 microarray software suite. Meth. Enzymol. 411, 134-193 (2006).

85 Cruz JA, Wishart DS: Applications of machine learning in cancer prediction and prognosis. Cancer Inform. 2, 59-77 (2006).

86 Michael A, Ball G, Quatan N et al.: Delayed disease progression after allogeneic cell vaccination in hormone-resistant prostate cancer and correlation with immunologic variables. Clin. Cancer Res. 11, 4469-4478 (2005).

87 Doreau A, Belot A, Bastid J et al.: Interleukin 17 acts in synergy with B cell-activating factor to influence B cell biology and the pathophysiology of systemic lupus erythematosus. Nat. Immunol. 10, 778-785 (2009).

88 Evans CFM, Galustian C, Bodman-Smith M, Dalgleish A, Kumar D: The effect of colorectal cancer upon host peripheral immune cell function. Colorectal Dis. 12, 561-569 (2010).

89 Ohtani K, Ohstuka Y, Ikuse T et al.: Increased mucosal expression of GATA-3 and STAT-4 in pediatric ulcerative colitis. Pediatr. Int. 52(4), 584-589 (2010).

90 Satyam A, Singh P, Badjatia N, Seth A, Sharma A: A disproportion of $\mathrm{T}(\mathrm{H}) 1 / \mathrm{T}(\mathrm{H}) 2$ cytokines with predominance of $\mathrm{T}(\mathrm{H}) 2$, in urothelial carcinoma of bladder. Urol. Oncol. DOI:10.1016/j.urolonc.2009.06.002 (2009) (Epub ahead of print).
91 Oliveira G, Xavier P, Murphy B et al.: Cytokine analysis of human renal allograft aspiration biopsy cultures supernatants predicts acute rejection. Nephrol. Dial. Transplant. 13, 417-422 (1998).

92 Zisakis A, Piperi C, Themistocleous MS et al:: Comparative analysis of peripheral and localised cytokine secretion in glioblastoma patients. Cytokine 39, 99-105 (2007).

93 Li H, Wang C, Yu J et al.: Dendritic cell-activated cytokine-induced killer cells enhance the anti-tumor effect of chemotherapy on non-small cell lung cancer in patients after surgery. Cytotherapy 11, 1076-1083 (2010).

94 Soleimani A, Berntsen A, Svane IM, Pedersen AE: Immune responses in patients with metastatic renal cell carcinoma treated with dendritic cells pulsed with tumor lysate. Scand. J. Immunol. 70, 481-489 (2009).

95 Gitlitz BJ, Belldegrun AS, Zisman A et al.: A pilot trial of tumor lysate-loaded dendritic cells for the treatment of metastatic renal cell carcinoma. J. Immunother. 26, 412-419 (2003).

96 Leonhartsberger N, Ramoner R, Putz T et al: : Antigen-independent immune responses after dendritic cell vaccination. Cancer Immunol. Immunother. 56, 897-903 (2007).

97 Ribas A, Glaspy JA, Lee Y et al: : Role of dendritic cell phenotype, determinant spreading, and negative costimulatory blockade in dendritic cell-based melanoma immunotherapy. J. Immunother. 27, 354-367 (2004).

98 Bladon J, Taylor PC: Early reduction in number of $\mathrm{T}$ cells producing proinflammatory cytokines, observed after extracorporeal photopheresis, is not linked to apoptosis induction. Transplant. Proc. 35, 1328-1332 (2003).

99 Pukhalsky AL, Shmarina GV, Bliacher MS et al: Cytokine profile after rubella vaccine inoculation: evidence of the immunosuppressive effect of vaccination. Mediators Inflamm. 12, 203-207 (2003).

\section{Websites}

101 International Society for Biological Therapy in Cancer (iSBTc) Initiatives www.isbtc.org/initiatives

102 NIST/SEMATECH e-Handbook of Statistical Methods www.itl.nist.gov/div898/handbook

103 Computer program to support analysis of polyfunctional ICS data http://exon.niaid.nih.gov/spice 